\title{
Speed of the Goldstone Sound Mode of an Atomic Fermi Gas Loaded on a Square Optical Lattice with a Non-Abelian Gauge Field in the Presence of a Zeeman Field
}

\author{
Zlatko Koinov (iD ${ }^{1}$ and Israel Chávez Villalpando ${ }^{2}$ \\ ${ }^{1}$ Department of Physics and Astronomy, University of Texas at San Antonio, San Antonio, TX 78249, USA \\ ${ }^{2}$ Instituto de Investigaciones en Materiales, Universidad Nacional Autónoma de México, Apartado Postal 20-364, \\ 01000 Mexico Distrito Federal, Mexico \\ Correspondence should be addressed to Zlatko Koinov; zlatko.koinov@utsa.edu
}

Received 15 December 2017; Revised 24 May 2018; Accepted 27 May 2018; Published 2 July 2018

Academic Editor: Oleg Derzhko

Copyright (C) 2018 Zlatko Koinov and Israel Chávez Villalpando. This is an open access article distributed under the Creative Commons Attribution License, which permits unrestricted use, distribution, and reproduction in any medium, provided the original work is properly cited.

\begin{abstract}
The speed of the Goldstone sound mode of a spin-orbit-coupled atomic Fermi gas loaded in a square optical lattice with a nonAbelian gauge field in the presence of a Zeeman field is calculated within the Gaussian approximation and from the Bethe-Salpeter equation in the generalized random phase approximation. It is found that (i) there is no sharp change of the slope of the Goldstone sound mode across the topological quantum phase transition point and (ii) the Gaussian approximation significantly overestimates the speed of sound of the Goldstone mode compared to the value provided by the BS formalism.
\end{abstract}

\section{Introduction}

It is well known that topological superfluids are new states of matter that can be observed in two-dimensional atomic Fermi gases with strong Rashba spin-orbit coupling (SOC), conventional s-wave pairing, and out-of-plane magnetic field (Zeeman field) which breaks time-reversal symmetry. In what follows, we study the speed of sound of a twocomponent pseudospin- $\uparrow, \downarrow$ fermionic gas loaded on a square optical lattice with a non-Abelian gauge field $\mathbf{A}=\left(\alpha \sigma_{y},-\alpha \sigma_{x}\right)$ in the presence of a Zeeman field, where $\alpha$ is independently tunable parameter, and $\sigma_{i}, i=x, y, z$ are the Pauli matrices. The external non-Abelian gauge field effectively creates a SOC in the gas. The pseudospin of atoms can couple with the Zeeman field and the orbital degrees of freedom of atoms, which gives rise to a topological quantum phase transition (TQPT) between gapped (topological trivial) and gapless (topological nontrivial) superfluid states. In other words, the spectrum of the Hamiltonian becomes gapless at some set of points in the momentum space. Due to absence of symmetry breaking across the TQPTs, it remains experimentally challenging to detect these phase transitions. It is experimentally possible to detect TQPTs by measuring the dynamic response of the bulk under an external force [1], by observing the edge modes $[2,3]$, or the momentum distributions of atoms across the phase transition boundary using a noise-correlation imaging [4] and momentum-resolved spectroscopy $[5,6]$.

Recent numerical calculations of the sound speed of atomic Fermi gases with s-wave attraction, synthetic Rashba SOC, and out-of-plane Zeeman field in two- and threedimensional free space [7-9] show sharp changes of the slope of the Goldstone sound mode across the phase transition points, and this can be used to detect TQPTs. Our goal is to study whether a similar behavior of the sound speed occurs in the lattice case if the SOC is created by the non-Abelian gauge field. We found that the sharp change in the slope does not exist in the presence of lattice geometry.

It is widely accepted among the cold-atom community that the system of a two-component Fermi gas with swave attraction in square optical lattice with an external non-Abelian gauge field (or a synthetic Rashba SOC) and out-of-plane Zeeman field can be described by a negativeU Hubbard model [10-26]. We restrict the discussion to the case of atoms confined to the lowest-energy band 
(single-band model). We assume that there are $M=N_{\uparrow}+N_{\downarrow}$ atoms distributed along $N$ sites, and the filling factor $f=$ $f_{\uparrow}+f_{\downarrow}=M / N$ is smaller than unity. The corresponding polarization is $P=\left(f_{\uparrow}-f_{\downarrow}\right) / f$. The Hamiltonian for an uniform system is $\widehat{H}=\widehat{H}_{0}+\widehat{H}_{U}$, where

$$
\widehat{H}_{0}=-\sum_{\langle i, j\rangle, \sigma, \sigma^{\prime}} \psi_{i, \sigma}^{\dagger} t_{i, j}^{\sigma \sigma^{\prime}} \psi_{j, \sigma^{\prime}}-\mu \sum_{i, \sigma} \widehat{n}_{i, \sigma}+\widehat{H}_{Z} .
$$

The out-of-plane Zeeman field is described by the term $\widehat{H}_{Z}$ :

$$
\widehat{H}_{Z}=h \sum_{i}\left(\psi_{i, \uparrow}^{\dagger}, \psi_{i, \downarrow}^{\dagger}\right) \sigma_{z}\left(\begin{array}{c}
\psi_{i, \uparrow} \\
\psi_{i, \downarrow}
\end{array}\right) .
$$

The Hubbard part of the Hamiltonian is

$$
\widehat{H}_{U}=-U \sum_{i} \widehat{n}_{i, \uparrow} \widehat{n}_{i, \downarrow}
$$

Here, $\mu$ is the chemical potential, and $\widehat{n}_{i, \sigma}=\psi_{i, \sigma}^{\dagger} \psi_{i, \sigma}$ is the density operator on site $i$. The Fermi operator $\psi_{i, \sigma}^{\dagger}=$ $(1 / N) \sum_{\mathbf{k}} \exp \left(-\imath \mathbf{k} \cdot \mathbf{r}_{i}\right) \psi_{\mathbf{k}, \sigma}^{\dagger}\left(\psi_{i, \sigma}=(1 / N) \sum_{\mathbf{k}} \exp \left(\imath \mathbf{k} \cdot \mathbf{r}_{i}\right) \psi_{\mathbf{k}, \sigma}\right)$ creates (destroys) a fermion on the lattice site $\mathbf{r}_{i}$ with pseudospin projection $\sigma$. The sum $\sum_{\langle i j\rangle}$ means sum over the nearest-neighbor sites of the $2 \mathrm{D}$ lattice. $t_{i, j}^{\sigma \sigma^{\prime}}=t_{i, j} \exp \left[-\imath \theta_{i, j}^{\sigma \sigma^{\prime}}\right]$ describes the hoping of the atoms between site $j$ and site $i$, and $\theta_{i, j}^{\sigma \sigma^{\prime}}=\int_{\mathbf{r}_{j}}^{\mathbf{r}_{i}} \mathbf{A} . d \mathbf{r}$ is accumulated via the Peierls substitution phase factor. The strength of the on-site Hubbard interaction is $U>0$, which corresponds to attractive interaction.

Hamiltonian (1) in a momentum representation assumes the form $\widehat{H}_{0}=\sum_{\mathbf{k}} \psi_{\mathbf{k}}^{\dagger}\left[\xi(\mathbf{k}) \sigma_{0}-h \sigma_{z}+J_{x}(\mathbf{k}) \sigma_{x}+J_{y}(\mathbf{k}) \sigma_{y}\right] \psi_{\mathbf{k}}$. Here, $\psi_{\mathbf{k}}^{\dagger}=\left(\psi_{\mathbf{k}, \uparrow}^{\dagger}, \psi_{\mathbf{k}, \downarrow}^{\dagger}\right), \xi(\mathbf{k})=\epsilon(\mathbf{k})-\mu, \epsilon(\mathbf{k})$ is the tight-binding energy, $\sigma_{0}$ is the identity matrix, and $J_{x, y}(\mathbf{k})$ characterize the SOC.

In the case of a square lattice the vectors $\mathbf{k}=\left(k_{x}, k_{y}\right)$ (in units $a^{-1}$ ) are restricted to be within the Brillouin zone, i.e., a square defined by the following four points $( \pm \pi / a, \pm \pi / a)$. The tight-binding energy and $J_{x, y}$ are given by

$$
\begin{aligned}
\epsilon(\mathbf{k}) & =-2 t \cos (\alpha)\left[\cos \left(k_{x}\right)+\cos \left(k_{y}\right)\right], \\
J_{x}(\mathbf{k}) & =-2 t \sin (\alpha) \sin \left(k_{y}\right), \\
J_{y}(\mathbf{k}) & =2 t \sin (\alpha) \sin \left(k_{x}\right),
\end{aligned}
$$

where $t$ is the strength of the nearest-neighbor hopping (in what follows, the lattice spacing $a$ and spacing $t$ are set to unity).

The opening or closing of the gap can be achieved by varying the tunable parameter $\alpha$ along with the Zeeman field. We first assume that the opening or closing of the gap is achieved by varying the parameter $\alpha$, while by changing the out-of-plane Zeeman field the polarization is kept fixed. We also discuss the second possibility, when one can close or open the gap by changing the Zeeman field while keeping the parameter $\alpha$ fixed. In this case the polarization changes during the transition from gapless to gapped superfluid states.
The single-particle and collective excitations of the system under consideration manifest themselves as poles of the single-particle and two-particle Green's functions, but the corresponding expressions for the Green's functions cannot be evaluated exactly because the interaction part of the Hubbard Hamiltonian is quartic in the fermion fields. The simplest way to solve this problem is to apply the so-called mean field decoupling of the quartic interaction. According to the standard mean field theory on the Hubbard Hamiltonian, the on-site interaction is decoupled (up to Hartree-Fock correction terms) as

$$
-U \widehat{n}_{i, \uparrow} \widehat{n}_{i, \downarrow} \approx \Delta\left(\psi_{i, \downarrow} \psi_{i, \uparrow}+\psi_{i, \uparrow}^{\dagger} \psi_{i, \downarrow}^{\dagger}+\frac{\Delta}{U}\right)
$$

where the order parameter $\Delta=-U\left\langle\psi_{i, \downarrow} \psi_{i, \uparrow}\right\rangle$ is a real number.

Instead of applying the mean field decoupling, we shall transform the quartic terms to a quadratic form by introducing a four-component boson field which mediates the interaction of fermions in the same manner as in quantum electrodynamics, where the photons mediate the interaction of electric charges. Green's functions are thermodynamic averages of the $\widehat{T}_{u}$-ordered products of field operators. The standard procedure for calculating Green's functions is to apply Wick's theorem. This enables us to evaluate the $\widehat{T}_{u}$-ordered products of field operators as perturbation expansions involving only wholly contracted field operators. These expansions can be summed formally to yield different equations of Green's functions. The main disadvantage of this procedure is that the validity of the equations must be verified diagram by diagram. For this reason, we shall use the method of Legendre transforms [27] of the generating functional for connected Green's functions to derive the Schwinger-Dyson $[28,29]$ (SD) equation $G^{-1}=G^{(0)-1}-\sum$ for the poles of the single-particle Green's function, $G$, and the Bethe-Salpeter $[30,31](B S)$ equation $\left[K^{(0)-1}-I\right] \Psi=0$ for the poles of the poles of the two-particle Green's function, $K$. Here, $G^{(0)}$ is the free single-particle propagator, $\Sigma$ is the fermion selfenergy, $I$ is the BS kernel, and the two-particle free propagator $K^{(0)}=G G$ is a product of two fully dressed single-particle Green's functions. The kernel of the BS equation is defined as a sum of the direct interaction, $I_{d}=\delta \Sigma^{F} / \delta G$, and the exchange interaction $I_{\text {exc }}=\delta \Sigma^{H} / \delta G$, where $\Sigma^{F}$ and $\Sigma^{H}$ are the Fock and the Hartree parts of the fermion self-energy $\Sigma$. Since the fermion self-energy depends on the two-particle Green's function, the positions of both poles must to be obtained by solving the SD and BS equations self-consistently. Instead of solving self-consistently the SD and BS equations, we shall employ the generalized random phase approximation (GRPA). In this approximation, the single-particle excitations are obtained in the mean field approximation, while the collective modes are obtained by solving the BS equation in which single-particle Green's functions are calculated in Hartree-Fock approximation, and the BS kernel is obtained by summing ladder and bubble diagrams.

\section{Functional-Integral Formalism}

2.1. Field-Theoretical Approach to the Hubbard Model. The functional-integral formulation of the Hubbard model 
requires the representation of the Hubbard interaction $-U \sum_{i} \widehat{n}_{i, \uparrow} \widehat{n}_{i, \downarrow}$ in terms of squares of fermion operators. This can be done by employing a certain Hubbard-Stratonovich transformation. This field-theoretical approach to the Hubbard Hamiltonian has already been used to describe the collective modes of ultracold ${ }^{6} \mathrm{Li}_{-}{ }^{40} \mathrm{~K}$ mixture in a square optical lattice (see [32]). Hamiltonian (1) in the present study has two more interactions. It is possible to include both the SOC and the Zeeman field into initial definition of the free fermion Green's function, and therefore most of the general equations in [32] remain unchanged. In particular, the general expressions for the SD and BS equations are the same as those without the SOC and the Zeeman field, but with more complicated fermion self-energy and BS kernel.

As it is well known, Green's functions in the functionalintegral approach are defined by means of the so-called generating functional with sources for the boson and fermion fields, but the corresponding functional integrals cannot be evaluated exactly because the interaction part of the Hubbard Hamiltonian is quartic in the Grassmann fermion fields. However, we can transform the quartic terms to a quadratic form by introducing a model system which consists of a fourcomponent boson field $A_{\alpha}(z)$ interacting with fermion fields $\widehat{\bar{\psi}}(y)=\widehat{\Psi}^{\dagger}(y) / \sqrt{2}$ and $\widehat{\psi}(x)=\widehat{\Psi}(x) / \sqrt{2}$, where

$$
\begin{aligned}
& \widehat{\Psi}(x)=\left(\begin{array}{c}
\psi_{\uparrow}(x) \\
\psi_{\downarrow}(x) \\
\psi_{\uparrow}^{\dagger}(x) \\
\psi_{\downarrow}^{\dagger}(x)
\end{array}\right), \\
& \widehat{\Psi}^{\dagger}(y)=\left(\psi_{\uparrow}^{\dagger}(y) \psi_{\downarrow}^{\dagger}(y) \psi_{\uparrow}(y) \psi_{\downarrow}(y)\right) .
\end{aligned}
$$

The symbol $\sum_{\omega_{m}}$ is used to denote $\beta^{-1} \sum_{m}$ (for fermion fields $\left.\omega_{m}=(2 \pi / \beta)(m+1 / 2) ; m=0, \pm 1, \pm 2, \ldots\right)$. is

Here $\alpha=1,2,3,4, x=\left(\mathbf{r}_{i}, u\right), y=\left(\mathbf{r}_{j}, u^{\prime}\right)$, and $z=$ $\left(\mathbf{r}_{k}, v\right)$ are composite variables, where $\mathbf{r}_{i}, \mathbf{r}_{j}$, and $\mathbf{r}_{k}$ are the lattice site vectors. According to imaginary-time (Matsubara) formalism the variables $0 \leq v, u, u^{\prime} \leq \beta=\left(k_{B} T\right)^{-1} ; k_{B}$ is the Boltzmann constant $\left(\hbar=k_{B}=1\right)$.

The field operators (6) allow us to define generalized single-particle Green's function by using a tensor product of these two matrices. The corresponding Green function, represented by a $4 \times 4$ matrix, includes all possible thermodynamic averages:

$$
\widehat{G}\left(x_{1} ; y_{2}\right)=-\left\langle\widehat{T}_{u}\left(\widehat{\Psi}\left(x_{1}\right) \otimes \widehat{\bar{\Psi}}\left(y_{2}\right)\right)\right\rangle .
$$

The action of the above-mentioned model system is assumed to be of the following form $S=S_{0}^{(F)}+$ $S_{0}^{(B)}+S^{(F-B)}$, where $S_{0}^{(F)}=\widehat{\bar{\psi}}(y) \widehat{G}^{(0)-1}(y ; x) \widehat{\psi}(x), S_{0}^{(B)}=$ $(1 / 2) A_{\alpha}(z) D_{\alpha \beta}^{(0)-1}\left(z, z^{\prime}\right) A_{\beta}\left(z^{\prime}\right), S^{(F-B)}=\widehat{\bar{\psi}}(y) \widehat{\Gamma}_{\alpha}^{(0)}(y, x$ । $z) \widehat{\psi}(x) A_{\alpha}(z)$. The fermion action $S_{0}^{(F)}$ corresponds to the Hamiltonian $\widehat{H}_{0}$. The corresponding inverse Green function of free fermions $\widehat{G}^{(0)-1}(y ; x)$ is given by the following $4 \times 4$ matrix:

$$
\begin{aligned}
& \widehat{G}^{(0)-1}(y ; x)=\sum_{\mathbf{k}, \omega_{m}} \exp \left[\imath \mathbf{k} \cdot\left(\mathbf{r}_{i}-\mathbf{r}_{i^{\prime}}\right)-\omega_{m}\left(u-u^{\prime}\right)\right] \\
& \cdot \widehat{G}^{(0)-1}\left(\mathbf{k}, \imath \omega_{m}\right) .
\end{aligned}
$$

The Fourier transform of noninteracting Green's function

$$
\widehat{G}^{(0)-1}\left(\mathbf{k}, \imath \omega_{m}\right)=\left(\begin{array}{cccc}
\imath \omega_{m}-\xi(\mathbf{k})-h & -\left(J_{x}(\mathbf{k})+\imath J_{y}(\mathbf{k})\right) & 0 & 0 \\
-\left(J_{x}(\mathbf{k})-\imath J_{y}(\mathbf{k})\right) & \imath \omega_{m}-\xi(\mathbf{k})+h & 0 & 0 \\
0 & 0 & \imath \omega_{m}+\xi(\mathbf{k})+h & -\left(J_{x}(\mathbf{k})-\imath J_{y}(\mathbf{k})\right) \\
0 & 0 & -\left(J_{x}(\mathbf{k})+\imath J_{y}(\mathbf{k})\right) & \imath \omega_{m}+\xi(\mathbf{k})-h
\end{array}\right)
$$

After including the Zeeman field and the SOC interaction into free fermion Green's function (9), one can perform the same steps as in [32] to come up with the following singleparticle Green function:

$$
\widehat{G}^{-1}(1 ; 2)=\left(\begin{array}{cccc}
G_{11}^{(0)-1} & G_{12}^{(0)-1} & 0 & -\Delta_{i_{1}, i_{2}} \\
G_{21}^{(0)-1} & G_{22}^{(0)-1} & \Delta_{i_{1}, i_{2}} & 0 \\
0 & \Delta_{i_{1}, i_{2}} & G_{33}^{(0)-1} & G_{31}^{(0)-1} \\
-\Delta_{i_{1}, i_{2}} & 0 & G_{41}^{(0)-1} & G_{44}^{(0)-1}
\end{array}\right)
$$

where $G_{i j}^{(0)-1}=G_{i j}^{(0)-1}\left(\mathbf{r}_{i_{1}}-\mathbf{r}_{i_{2}} ; u_{1}-u_{2}\right)$, and $\Delta_{i_{1}, i_{2}} \equiv \Delta \delta\left(\mathbf{r}_{i_{1}}-\right.$ $\mathbf{r}_{i_{2}}$ ), where $\Delta$ is a constant in space. Physically, it describes a superfluid state of Cooper pairs with zero momentum when the pairing is only between atoms with equal and opposite momenta.

2.2. Mean Field Approximation. The Fourier transform of zero-temperature single-particle Green's function (10) in the mean field approximation is 


$$
\widehat{G}_{M F}\left(\mathbf{k}, \imath \omega_{m}\right)=\left(\begin{array}{cccc}
\imath \omega_{m}-\xi(\mathbf{k})-h & -\left(J_{x}(\mathbf{k})+\imath J_{y}(\mathbf{k})\right) & 0 & \Delta \\
-\left(J_{x}(\mathbf{k})-\imath J_{y}(\mathbf{k})\right) & \imath \omega_{m}-\xi(\mathbf{k})+h & -\Delta & 0 \\
0 & -\Delta & \imath \omega_{m}+\xi(\mathbf{k})+h & -\left(J_{x}(\mathbf{k})-\imath J_{y}(\mathbf{k})\right) \\
\Delta & 0 & -\left(J_{x}(\mathbf{k})+\imath J_{y}(\mathbf{k})\right) & \imath \omega_{m}+\xi(\mathbf{k})-h
\end{array}\right)^{-1}
$$

The matrix elements $\widehat{G}_{n_{1} n_{2}}^{M F}\left(\mathbf{k}, \imath \omega_{m}\right)\left(n_{1}, n_{2}=1,2,3,4\right)$ of $\widehat{G}_{M F}(\mathbf{k}, \omega)$ are given by

$$
\begin{aligned}
G_{n_{1} n_{2}}^{M F}(\mathbf{k}, \omega)= & \frac{A_{n_{1} n_{2}}(\mathbf{k})}{\omega-\omega(\mathbf{k})+\imath 0^{+}}+\frac{B_{n_{1} n_{2}}(\mathbf{k})}{\omega+\omega(\mathbf{k})-\imath 0^{+}} \\
& +\frac{C_{n_{1} n_{2}}(\mathbf{k})}{\omega-\Omega(\mathbf{k})+\imath 0^{+}}+\frac{D_{n_{1} n_{2}}(\mathbf{k})}{\omega+\Omega(\mathbf{k})-\imath 0^{+}},
\end{aligned}
$$

where the corresponding expressions for $A_{n_{1} n_{2}}(\mathbf{k}), B_{n_{1} n_{2}}(\mathbf{k})$, $C_{n_{1} n_{2}}(\mathbf{k})$, and $D_{n_{1} n_{2}}(\mathbf{k})$ can be obtained by inverting matrix (11) and setting $T \longrightarrow 0$.

For a fixed filing factor $f=f_{\uparrow}+f_{\downarrow}$ and at a given Zeeman field $h$, the chemical potential $\mu$ and the gap $\Delta$ have to be determined by solving the mean field number and gap equations:

$$
\begin{aligned}
& f=1-\sum_{\mathbf{k}}\left[\frac{1}{2}-F(\Omega(\mathbf{k}))\right] \frac{\xi(\mathbf{k})}{\Omega(\mathbf{k})} \\
& \cdot\left(1+\frac{S(\mathbf{k})+h^{2}}{\sqrt{\left(S(\mathbf{k})+h^{2}\right) \xi^{2}(\mathbf{k})+h^{2} \Delta^{2}}}\right) \\
& -\sum_{\mathbf{k}}\left[\frac{1}{2}-F(\omega(\mathbf{k}))\right] \frac{\xi(\mathbf{k})}{\omega(\mathbf{k})} \\
& \cdot\left(1-\frac{S(\mathbf{k})+h^{2}}{\sqrt{\left(S(\mathbf{k})+h^{2}\right) \xi^{2}(\mathbf{k})+h^{2} \Delta^{2}}}\right), \\
& \frac{1}{U}=\sum_{\mathbf{k}}\left[\frac{1}{2}-F(\Omega(\mathbf{k}))\right] \frac{1}{2 \Omega(\mathbf{k})} \\
& \cdot\left(1+\frac{h^{2}}{\sqrt{\left(S(\mathbf{k})+h^{2}\right) \xi^{2}(\mathbf{k})+h^{2} \Delta^{2}}}\right) \\
& +\sum_{\mathbf{k}}\left[\frac{1}{2}-F(\omega(\mathbf{k}))\right] \frac{1}{2 \omega(\mathbf{k})} \\
& \cdot\left(1-\frac{h^{2}}{\sqrt{\left(S(\mathbf{k})+h^{2}\right) \xi^{2}(\mathbf{k})+h^{2} \Delta^{2}}}\right) .
\end{aligned}
$$

Here, $S(\mathbf{k})=J_{x}^{2}(\mathbf{k})+J_{y}^{2}(\mathbf{k}), F(\omega)$ is the Fermi-Dirac distribution function, and the following notations have been introduced:
$\Omega(\mathbf{k})$

$$
=\sqrt{S(\mathbf{k})+\xi^{2}(\mathbf{k})+\Delta^{2}+h^{2}+2 \sqrt{\left(S(\mathbf{k})+h^{2}\right) \xi^{2}(\mathbf{k})+h^{2} \Delta^{2}}},
$$

$\omega(\mathbf{k})$

$$
=\sqrt{S(\mathbf{k})+\xi^{2}(\mathbf{k})+\Delta^{2}+h^{2}-2 \sqrt{\left(S(\mathbf{k})+h^{2}\right) \xi^{2}(\mathbf{k})+h^{2} \Delta^{2}}} .
$$

The number equation (13) follows from the definitions of the filing factors $f_{\uparrow,(\downarrow)}=\sum_{\mathbf{k}} \sum_{\imath \omega_{m}} G_{11,(22)}^{M F}\left(\mathbf{k}, \imath \omega_{m}\right)$ through corresponding mean field Green's functions. It is known that the gap equation can be obtained by minimizing the mean field Helmholtz free energy with respect to $\Delta$.

As we mentioned before, in order to keep the polarization fixed, one has to adjust the corresponding Zeeman field. This means that $\mu, \Delta$, and $h$ have to be calculated by solving the number and the gap equations, along with the following equation:

$$
\begin{aligned}
& f_{\downarrow}-f_{\uparrow}=\sum_{\mathbf{k}}\left[\frac{1}{2}-F(\Omega(\mathbf{k}))\right] \\
& \cdot \frac{h}{\Omega(\mathbf{k})}\left(1+\frac{\xi^{2}(\mathbf{k})+\Delta^{2}}{\sqrt{\left(S(\mathbf{k})+h^{2}\right) \xi^{2}(\mathbf{k})+h^{2} \Delta^{2}}}\right) \\
& +\sum_{\mathbf{k}}\left[\frac{1}{2}-F(\omega(\mathbf{k}))\right] \\
& \cdot \frac{h}{\omega(\mathbf{k})}\left(1-\frac{\xi^{2}(\mathbf{k})+\Delta^{2}}{\sqrt{\left(S(\mathbf{k})+h^{2}\right) \xi^{2}(\mathbf{k})+h^{2} \Delta^{2}}}\right) .
\end{aligned}
$$

Another interesting feature is that because of the SOC term in Hamiltonian (1) the pairing field contains both singlet and triplet component. The singlet $\Phi_{\downarrow \uparrow}(\mathbf{k})=-\Phi_{\uparrow \downarrow}(\mathbf{k})=$ $\left\langle\psi_{\mathbf{k} \downarrow} \psi_{-\mathbf{k} \uparrow}\right\rangle$ and triplet $\Phi_{\uparrow \uparrow}(\mathbf{k})=\left\langle\psi_{\mathbf{k} \uparrow} \psi_{-\mathbf{k} \uparrow}\right\rangle, \Phi_{\downarrow \downarrow}(\mathbf{k})=$ $\left\langle\psi_{\mathbf{k} \downarrow} \psi_{-\mathbf{k} \downarrow}\right\rangle$ amplitudes, obtained by means of Green's function elements $G_{23}^{M F}, G_{13}^{M F}$, and $G_{24}^{M F}$, are

$$
\begin{gathered}
\Phi_{\downarrow \uparrow}(\mathbf{k})=\frac{\Delta\left[1 / 2-f_{F}(\Omega(\mathbf{k}))\right]}{2 \Omega(\mathbf{k})}[1 \\
\left.+\frac{h^{2}}{\sqrt{S(\mathbf{k}) \xi^{2}(\mathbf{k})+h^{2}\left[\Delta^{2}+\xi^{2}(\mathbf{k})\right]}}\right]
\end{gathered}
$$




$$
\begin{aligned}
& +\frac{\Delta\left[1 / 2-f_{F}(\omega(\mathbf{k}))\right]}{2 \omega(\mathbf{k})}[1 \\
& \left.-\frac{h^{2}}{\sqrt{S(\mathbf{k}) \xi^{2}(\mathbf{k})+h^{2}\left[\Delta^{2}+\xi^{2}(\mathbf{k})\right]}}\right]
\end{aligned}
$$

$\Phi_{\uparrow \uparrow}(\mathbf{k})$

$$
\begin{aligned}
& =\frac{\Delta J(\mathbf{k})[h-\xi(\mathbf{k})]}{2 \sqrt{S(\mathbf{k}) \xi^{2}(\mathbf{k})+h^{2}\left[\Delta^{2}+\xi^{2}(\mathbf{k})\right]}}\left[\frac{1 / 2-f_{F}(\Omega(\mathbf{k}))}{\Omega(\mathbf{k})}\right. \\
& \left.-\frac{1 / 2-f_{F}(\omega(\mathbf{k}))}{\omega(\mathbf{k})}\right] ;
\end{aligned}
$$

$\Phi_{\downarrow \downarrow}(\mathbf{k})$

$$
\begin{aligned}
& =\frac{\Delta J^{*}(\mathbf{k})[h+\xi(\mathbf{k})]}{2 \sqrt{S(\mathbf{k}) \xi^{2}(\mathbf{k})+h^{2}\left[\Delta^{2}+\xi^{2}(\mathbf{k})\right]}}\left[\frac{1 / 2-f_{F}(\Omega(\mathbf{k}))}{\Omega(\mathbf{k})}\right. \\
& \left.-\frac{1 / 2-f_{F}(\omega(\mathbf{k}))}{\omega(\mathbf{k})}\right] .
\end{aligned}
$$

With the help of the pairing amplitudes, one can calculate the total condensate fraction $f_{c}=f_{s}+f_{t r}$, where $f_{s}$ and $f_{t r}$ are the singlet and the triplet contributions, correspondingly. At zero temperature, we obtain

$$
\begin{aligned}
& f_{s}=\frac{2}{N} \sum_{\mathbf{k}}\left|\Phi_{\downarrow \uparrow}(\mathbf{k})\right|^{2}=\frac{\Delta^{2}}{8 N} \sum_{\mathbf{k}}\left[\frac{1}{\Omega(\mathbf{k})}(1\right. \\
& \left.+\frac{h^{2}}{\sqrt{S(\mathbf{k}) \xi^{2}(\mathbf{k})+h^{2}\left[\Delta^{2}+\xi^{2}(\mathbf{k})\right]}}\right)+\frac{1}{\omega(\mathbf{k})}(1 \\
& \left.\left.-\frac{h^{2}}{\sqrt{S(\mathbf{k}) \xi^{2}(\mathbf{k})+h^{2}\left[\Delta^{2}+\xi^{2}(\mathbf{k})\right]}}\right)\right]^{2}, \\
& f_{t r}=\frac{1}{N} \sum_{\mathbf{k}}\left(\left|\Phi_{\uparrow \uparrow}(\mathbf{k})\right|^{2}+\left|\Phi_{\downarrow \downarrow}(\mathbf{k})\right|^{2}\right)=\frac{\Delta^{2}}{8 N} \\
& \cdot \sum_{\mathbf{k}} \frac{S(\mathbf{k})\left[h^{2}+\xi^{2}(\mathbf{k})\right]}{S(\mathbf{k}) \xi^{2}(\mathbf{k})+h^{2}\left[\Delta^{2}+\xi^{2}(\mathbf{k})\right]}\left[\frac{1}{\Omega(\mathbf{k})}\right. \\
& \left.-\frac{1}{\omega(\mathbf{k})}\right]^{2} \text {. }
\end{aligned}
$$

2.3. The Bethe-Salpeter Equation for the Collective Excitations in the Generalized Random Phase Approximation. The spectrum of the collective modes will be obtained by solving the BS equation in the GRPA. As we have already mentioned, the kernel of the BS equation is a sum of the direct $I_{d}=$ $\delta \Sigma^{F} / \delta G$ and exchange $I_{\text {exc }}=\delta \Sigma^{H} / \delta G$ interactions, written as derivatives of the Fock and the Hartree parts of the selfenergy. Thus, in the GRPA the corresponding equation for the BS amplitude $\Psi_{n_{2}, n_{1}}^{\mathbf{Q}}$ is given by (see [32])

$$
\begin{aligned}
& \Psi_{n_{2} n_{1}}^{\mathbf{Q}}=K^{(0)}\left(\begin{array}{lll}
n_{1} & n_{3} & \mid \omega(\mathbf{Q})) \\
n_{2} & n_{4} & \mid
\end{array}\right] I_{d}\left(\begin{array}{ll}
n_{3} & n_{5} \\
n_{4} & n_{6}
\end{array}\right) \\
& \left.\quad+I_{\text {exc }}\left(\begin{array}{ll}
n_{3} & n_{5} \\
n_{4} & n_{6}
\end{array}\right)\right] \underset{n_{6}, n_{5}}{\mathbf{Q}},
\end{aligned}
$$

where $I_{d}\left(\begin{array}{ll}n_{1} & n_{3} \\ n_{2} & n_{4}\end{array}\right)=-\Gamma_{\alpha}^{(0)}\left(n_{1}, n_{3}\right) D_{\alpha \beta}^{(0)} \Gamma_{\beta}^{(0)}\left(n_{4}, n_{2}\right)$ and $I_{\text {exc }}\left(\begin{array}{ll}n_{1} & n_{3} \\ n_{2} & n_{4}\end{array}\right)=(1 / 2) \Gamma_{\alpha}^{(0)}\left(n_{1}, n_{2}\right) D_{\alpha \beta}^{(0)} \Gamma_{\beta}^{(0)}\left(n_{4}, n_{3}\right)$ are the direct and the exchange interactions, correspondingly. The two-particle propagator $K^{(0)}$ in the GRPA is defined as follows:

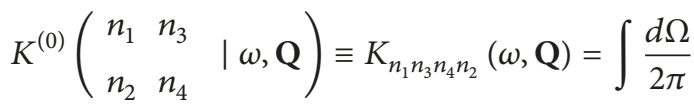

$$
\begin{aligned}
& \cdot \int \frac{d^{2} \mathbf{k}}{(2 \pi)^{2}} G_{n_{1} n_{3}}^{M F}(\mathbf{k}+\mathbf{Q}, \Omega+\omega(\mathbf{Q})) G_{n_{4} n_{2}}^{M F}(\mathbf{k}, \Omega) \\
& =\int \frac{d^{2} \mathbf{k}}{(2 \pi)^{2}}\left[\frac{A_{n_{1} n_{3}}(\mathbf{k}+\mathbf{Q}) B_{n_{4} n_{2}}(\mathbf{k})}{\omega-(\omega(\mathbf{k}+\mathbf{Q})+\omega(\mathbf{k}))}\right. \\
& -\frac{B_{n_{1} n_{3}}(\mathbf{k}+\mathbf{Q}) A_{n_{4} n_{2}}(\mathbf{k})}{\omega+(\omega(\mathbf{k}+\mathbf{Q})+\omega(\mathbf{k}))} \\
& +\frac{C_{n_{1} n_{3}}(\mathbf{k}+\mathbf{Q}) D_{n_{4} n_{2}}(\mathbf{k})}{\omega-(\Omega(\mathbf{k}+\mathbf{Q})+\Omega(\mathbf{k}))} \\
& -\frac{D_{n_{1} n_{3}}(\mathbf{k}+\mathbf{Q}) C_{n_{4} n_{2}}(\mathbf{k})}{\omega+(\Omega(\mathbf{k}+\mathbf{Q})+\Omega(\mathbf{k}))} \\
& +\frac{A_{n_{1} n_{3}}(\mathbf{k}+\mathbf{Q}) D_{n_{4} n_{2}}(\mathbf{k})}{\omega-(\omega(\mathbf{k}+\mathbf{Q})+\Omega(\mathbf{k}))} \\
& -\frac{B_{n_{1} n_{3}}(\mathbf{k}+\mathbf{Q}) C_{n_{4} n_{2}}(\mathbf{k})}{\omega+(\omega(\mathbf{k}+\mathbf{Q})+\Omega(\mathbf{k}))} \\
& +\frac{C_{n_{1} n_{3}}(\mathbf{k}+\mathbf{Q}) B_{n_{4} n_{2}}(\mathbf{k})}{\omega-(\Omega(\mathbf{k}+\mathbf{Q})+\omega(\mathbf{k}))} \\
& \left.-\frac{D_{n_{1} n_{3}}(\mathbf{k}+\mathbf{Q}) A_{n_{4} n_{2}}(\mathbf{k})}{\omega+(\Omega(\mathbf{k}+\mathbf{Q})+\omega(\mathbf{k}))}\right] \text {. }
\end{aligned}
$$

The BS equation (21) can be written in the matrix form as $(\widehat{I}+$ $U \widehat{Z}) \widehat{\Psi}=0$, where $\widehat{I}$ is the unit matrix, the matrix $\widehat{Z}$ is a $16 \times 16$ matrix, and the transposed matrix of $\widehat{\Psi}$ is given by

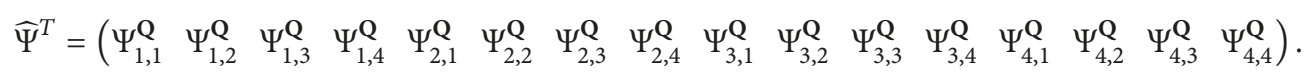


At a given vector $\mathbf{Q}$ from the Brillouin zone, the collective excitation spectrum $\omega(\mathbf{Q})$ is obtained by the condition $\operatorname{det} \mid \widehat{I}+$ $U \widehat{M}(\omega(\mathbf{Q}), \mathbf{Q})) \mid=0$ to have a nontrivial solution of the $\mathrm{BS}$ equation. By applying simple matrix algebra, the above secular $16 \times 16$ determinant can be simplified to a $10 \times 10$ BS determinant $\operatorname{det}|B S(\omega(\mathbf{Q}), \mathbf{Q})|=0$, where the BS matrix is given by

$$
B S(\omega, \mathbf{Q})=\left(\begin{array}{ll}
A_{2 \times 2}(\omega, \mathbf{Q}) & B_{2 \times 8}(\omega, \mathbf{Q}) \\
C_{8 \times 2}(\omega, \mathbf{Q}) & D_{8 \times 8}(\omega, \mathbf{Q})
\end{array}\right) .
$$

The elements $A_{i, j}, B_{i, j}, C_{i, j}$, and $D_{i, j}$ of the blocks $A_{2 \times 2}(\omega, \mathbf{Q})$, $B_{2 \times 8}(\omega, \mathbf{Q}), C_{8 \times 2}(\omega, \mathbf{Q})$, and $D_{8 \times 8}(\omega, \mathbf{Q})$ are given in the Appendix in terms of the propagators $K_{n_{1} n_{3} n_{4} n_{2}}(\omega, \mathbf{Q})$.

In contrast to our functional-integral formalism, one can use the Hubbard-Stratonovich transformation to introduce the energy gap as an order parameter field. In this approach, one can integrate out the fermion fields and to arrive at an effective action. The next steps are to consider the state which corresponds to the saddle point of the effective action and to write the effective action as a series in powers of the fluctuations and their derivatives. The exact result can be obtained by explicitly calculating the terms up to second order in the fluctuations and their derivatives. This approximation is called the Gaussian approximation. Within this approximation, the collective excitation spectrum $\omega(\mathbf{Q})$ is defined by the $2 \times 2$ Gaussian secular determinant

$$
\operatorname{det}\left|A_{2 \times 2}(\omega(\mathbf{Q}), \mathbf{Q})\right|=0,
$$

while within the BS formalism the secular determinant is more complicated $((\omega(\mathbf{Q}), \mathbf{Q})$ dependence is understood):

$$
\operatorname{det}\left|A_{2 \times 2}-B_{2 \times 8} \cdot D_{8 \times 8}^{-1} \cdot C_{8 \times 2}\right|=0
$$

\section{Numerical Results}

There are various mean field quantities of physical interest, such as the chemical potential, the pairing gap, the singlet and triplet pairing amplitudes, and the singlet and triplet condensate fractions. We focus on the zero-temperature case assuming two different filling factors of $f=0.4$ and $f=0.6$. The strength of the attractive interaction is $U / t=4$, and for both filling factors the polarization is fixed to be $P=0.48$.

In Figure 1, we plot the chemical potential $\mu$, the gap $\Delta$, and the corresponding Zeeman field $h$, as functions of the SOC parameter $\alpha$, obtained by solving the mean field equations (13)-(17). In Figure 2 we have shown the singlet and the triplet condensate fractions, as functions of the SOC parameter $\alpha$, obtained by solving the mean field equations along with definitions (19) and (20). It can be seen that at the value $\alpha=\pi / 2$, where $\xi(\mathbf{k})=-\mu$ and $S(\mathbf{k})=4 t^{2}\left[\sin ^{2}\left(k_{x}\right)+\right.$ $\left.\sin ^{2}\left(k_{y}\right)\right]$, the chemical potential has a minimum, while the gap and the Zeeman field reach their maximum values. As expected, the singlet and triplet condensate fraction-graphs have maximum at the same $\alpha$ as the gap.

Next, we have calculated the speed of sound as a function of the SOC parameter $\alpha$. The sound speed $c$ is proportional to the slope $u$ of the Goldstone sound mode $c=u a t / \hbar$. The slope
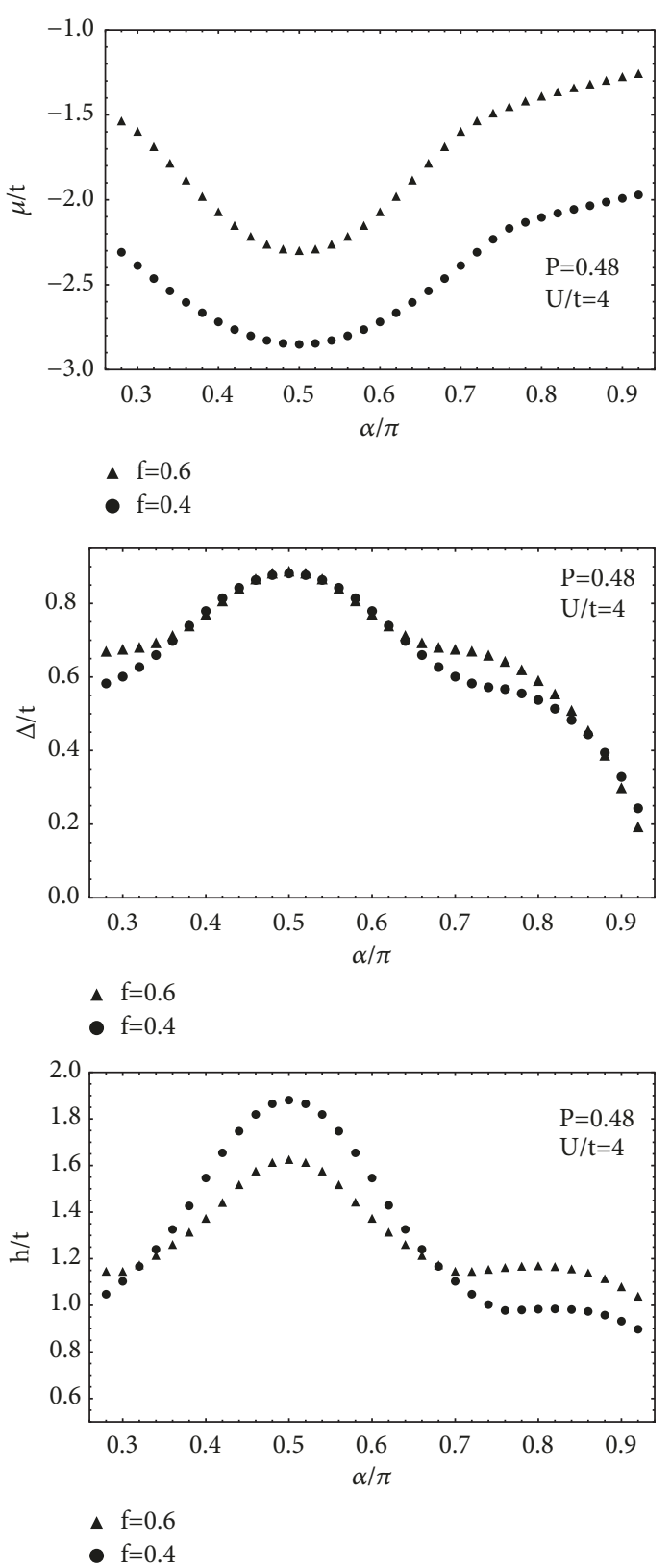

FIGURE 1: Chemical potential $\mu$, pairing gap $\Delta$, and the Zeeman field $h$ of a Fermi gas in a square optical lattice subject to a non-Abelian gauge field $\mathbf{A}=\left(\alpha \sigma_{y},-\alpha \sigma_{x}\right)$. The graphs are obtained by solving the mean field equations (13)-(17) using two different filling factors: $f=0.4$ and $f=0.6$. The on-site attractive strength is $U=4 t$, and the polarization is $P=0.48$.

$u$ has been numerically obtained using the collective-mode dispersion $\omega(\mathbf{Q})$ for small wave vectors in $Q_{x}$-direction.

It is clear that the single-particle dispersion, given by (16), becomes precisely zero at some special points $\mathbf{k}_{0}$ in momentum space satisfying the condition $S\left(\mathbf{k}_{0}\right)=0$ when $h=\sqrt{\left(\epsilon\left(\mathbf{k}_{0}\right)-\mu\right)^{2}+\Delta^{2}}$. The condition $S\left(\mathbf{k}_{0}\right)=0$ is met at the following points: $(0,0),(0, \pm \pi),( \pm \pi, 0)$, and $( \pm \pi, \pm \pi)$. For $U / t=4, P=0.48$, and a filling factor $f=0.4$, we have two points at which the single-particle dispersion 

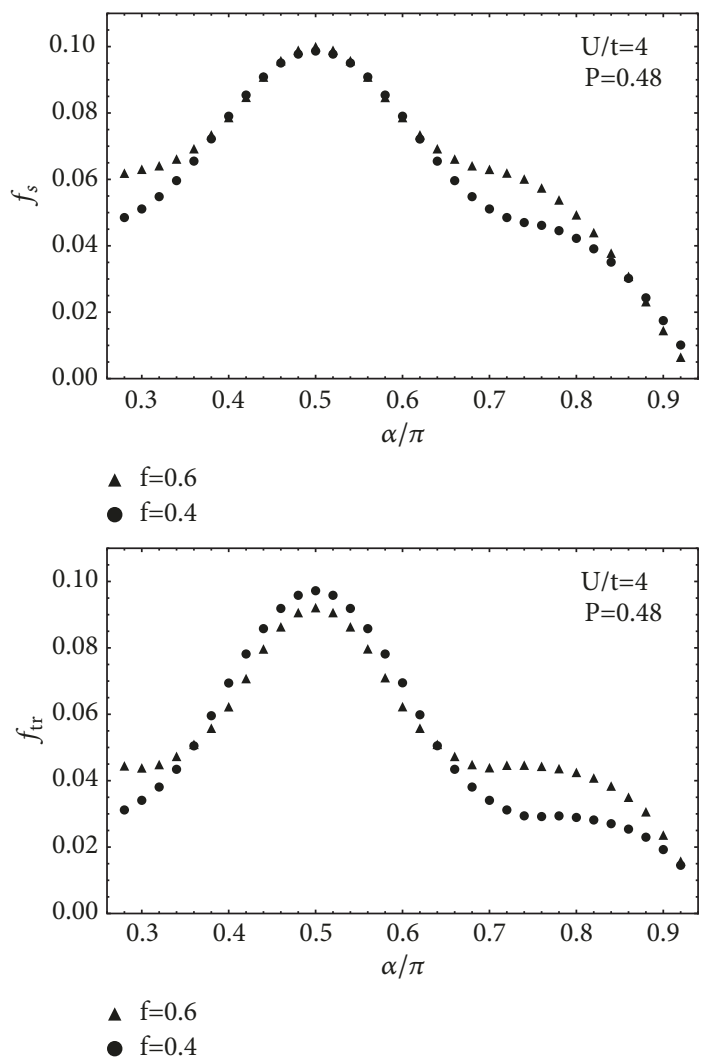

FIgURE 2: The singlet $f_{s}$ and the triplet $f_{\text {tr }}$ contributions to the total condensed fraction $f=f_{s}+f_{t r}$ of a Fermi gas in a square optical lattice subject to a non-Abelian gauge field $\mathbf{A}=\left(\alpha \sigma_{y},-\alpha \sigma_{x}\right)$. The graphs are obtained by solving the mean field equations along with definitions (19) and (20). The system parameters are the same as in Figure 1.

$\omega\left(\mathbf{k}_{0,1}\right)=0$. The first one is at the center of the Brillouin zone $\mathbf{k}_{0}=(0,0)$, and $\omega\left(\mathbf{k}_{0}\right)=0$ for $\alpha / \pi$ between 0.39 and 0.40 . At the second point, $\mathbf{k}_{1}=(\pi, \pi)$, we found also the existence of opening and closing of a gap in momentum space when $0.61<\alpha / \pi<0.62$. Therefore, at both points one should have a TQPT between superfluid states with different topologies. During the TQPT the different phases of matter are not characterized by an order parameter, but rather an integer number, the Chern number, describing the system as a whole. This is because the different phases across the TQPT are characterized by different topologies of Fermi surface instead of being classified by different symmetries.

In Figure 3, we have shown the slope calculated by using the Gaussian and the BS secular determinants (25) and (26), correspondingly. It is worth mentioning the significant difference between the slope obtained within the Gaussian approximation and the slope obtained by the BS secular determinant. The arrows show the points where the opening and closing of a gap take place. As can be seen, the sharp change in the slope does not exist in the presence of a nonAbelian gauge field.

The boundaries for the TQPT in [7-9] were accessed by varying the Zeeman filed at a constant strength of the

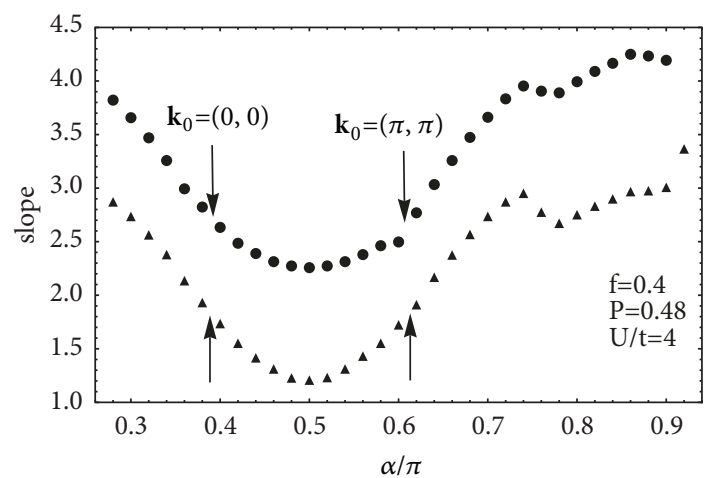

$\triangle$ BS

- Gauss

Figure 3: The slope of the Goldstone sound mode of a Fermi gas in a square optical lattice subject to a non-Abelian gauge field $\mathbf{A}=$ $\left(\alpha \sigma_{y},-\alpha \sigma_{x}\right)$. The graphs are obtained by applying the Gaussian and the BS secular determinants. The system parameters are the same as in Figure 1. The arrows show the values of $\alpha$ for which the opening and closing of a gap take place.

synthetic Rashba SOC. It was found that on a topological trivial side, where $h<h_{c}=\sqrt{\left(\epsilon\left(\mathbf{k}_{0}\right)-\mu\right)^{2}+\Delta^{2}}$, the slope is suppressed by the Zeeman field, while in the nontrivial side, where $h>h_{c}=\sqrt{\left(\epsilon\left(\mathbf{k}_{0}\right)-\mu\right)^{2}+\Delta^{2}}$, the slope is enhanced by the Zeeman field. As mentioned in $[7,8]$, this is quite unusual since we generally expect that the superfluidity should be suppressed by the Zeeman field.

We have fixed the non-Abelian parameter $\alpha=0.38 \pi$, and we have accessed the opening and the closing of a gap in momentum space at the point $\mathbf{k}_{0}=(0,0)$ by varying the Zeeman filed. To do this, first we used (13)-(14) to calculate numerically the chemical potential and the gap as functions of the Zeeman field. The results are shown in Figure 4. The TQPT takes place for $h_{c} / t=1.39$ and $h_{c} / t=1.49$. The insert shows the behavior of the slope close to $h_{c} / t=1.49$. We found that there is no sharp change in the slope across the two TQPT points.

\section{Discussion}

In this paper, we are concerned with the question which naturally arises here as to whether there is a possibility of detecting TQPTs by monitoring the behavior of slope of the sound mode if a non-Abelian gauge field is used instead of a synthetic Rashba SOC. The answer is not trivial because the non-Abelian gauge field is taken into account via the Peierls substitution. As a result, the tight-binding energy $\epsilon(\mathbf{k})=$ $-2 t \cos (\alpha)\left[\cos \left(k_{x}\right)+\cos \left(k_{y}\right)\right]$ does depend on the parameter $\alpha$. The second difference is that the nondiagonal part of $\theta_{i, j}^{\sigma \sigma^{\prime}}$, i.e., $\sigma \neq \sigma^{\prime}$, describes the spin-flipped hopping between site $i$ and site $j$. In the case of a synthetic Rashba SOC we do not have spin-flipped hopping, and the hopping to the nearestneighbor site does not depend on the strength of Rashba SOC. 

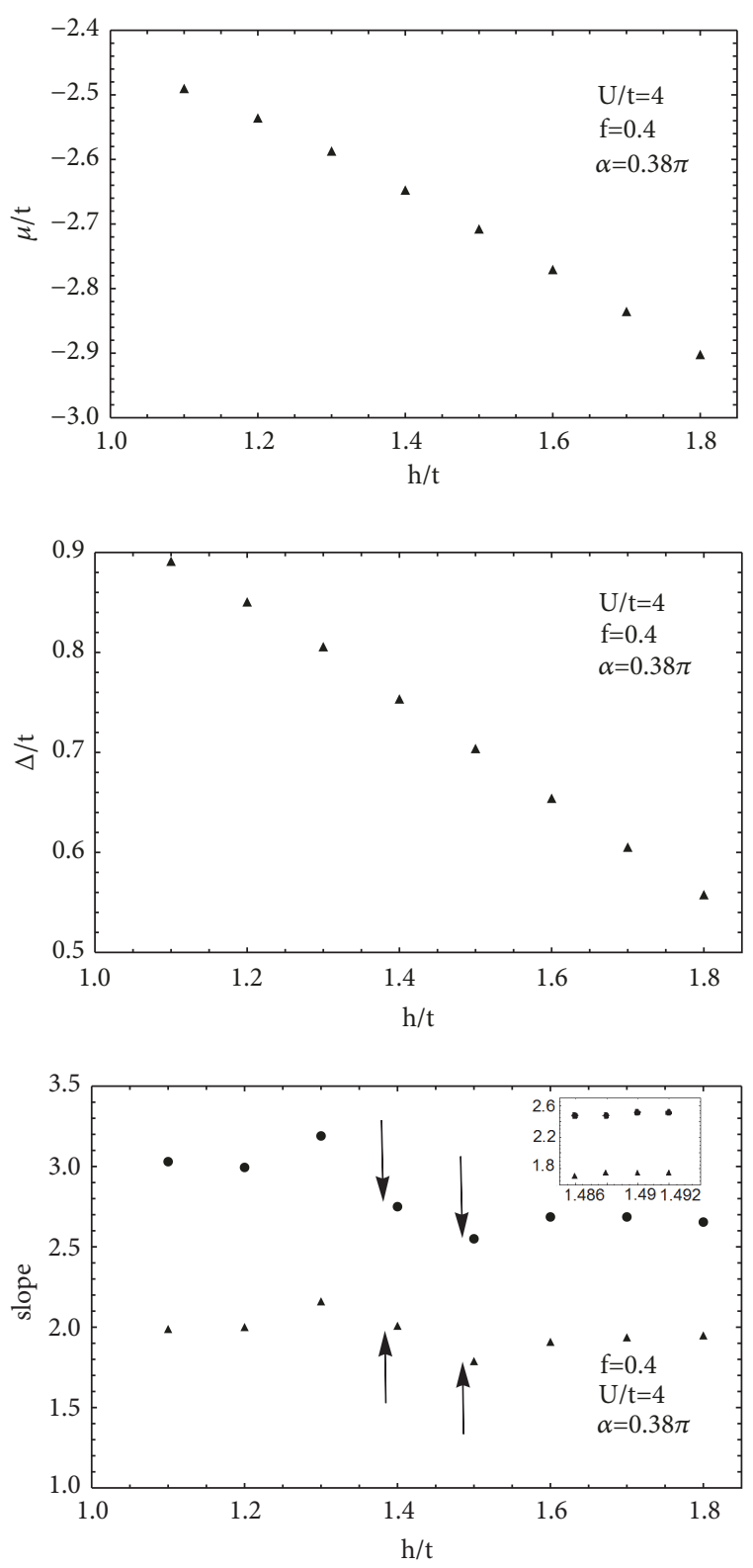

- Gauss

- BS

FIgURE 4: Chemical potential $\mu$, pairing gap $\Delta$, and the slope of the Goldstone sound mode of a Fermi gas in a square optical lattice subject to a non-Abelian gauge field with $\alpha=0.38 \pi$ as a function of the Zeeman field $h$. The chemical potential and the gap are obtained by solving the mean field number and gap equations (13)-(14). The arrows show the values of the Zeeman field for which the opening and closing of a gap take place. The insert shows the slope close to $h_{c} / t=1.49$.

In summary, we have derived the BS equation in the GRPA for the collective excitation energy of a Fermi gas loaded on a square optical lattice with a non-Abelian gauge field in the presence of a Zeeman field. To the best of our knowledge, there is no other calculation of the speed of sound in a lattice geometry as a function of the nonAbelian parameter with which we can make comparisons.
According to our numerical calculations, there is no sharp change of the slope of the Goldstone sound mode across the phase transition point. It is found that the Gaussian approximation significantly overestimates the speed of sound of the Goldstone mode compared to the value obtained within the BS formalism.

\section{Appendix}

\section{Blocks of the BS Matrix}

The two-particle propagator in BS equation (21) is defined as

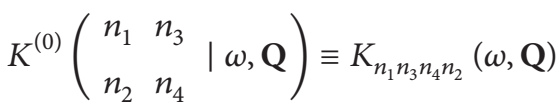

$$
\begin{aligned}
& =\sum_{\mathbf{k}} \int \frac{d \Omega}{2 \pi} G_{n_{1} n_{3}}^{M F}(\mathbf{k}+\mathbf{Q}, \Omega+\omega) G_{n_{4} n_{2}}^{M F}(\mathbf{k}, \Omega) \text {. }
\end{aligned}
$$

The elements $A_{i, j}, B_{i, j}, C_{i, j}$, and $D_{i, j}$ of the blocks $A_{2 \times 2}(\omega, \mathbf{Q}), B_{2 \times 8}(\omega, \mathbf{Q}), C_{8 \times 2}(\omega, \mathbf{Q})$, and $D_{8 \times 8}(\omega, \mathbf{Q})$ are given in terms of the propagators $K_{n_{1} n_{3} n_{4} n_{2}}(\omega, \mathbf{Q})$ as follows:

$$
\begin{aligned}
& A_{1,1}=1+\frac{U_{0}}{2}\left(K_{1144}+K_{2233}-K_{1234}-K_{2143}\right), \\
& A_{1,2}=\frac{U_{0}}{2}\left(K_{1234}+K_{2413}-K_{1414}-K_{2323}\right), \\
& A_{2,1}=\frac{U_{0}}{2}\left(K_{3142}+K_{4231}-K_{3232}-K_{4141}\right), \\
& A_{2,2}=1+\frac{U_{0}}{2}\left(K_{3322}+K_{4411}-K_{3412}-K_{4321}\right), \\
& B_{1,1}=\frac{U_{0}}{2}\left(K_{1124}-K_{2123}\right), \\
& B_{1,2}=\frac{U_{0}}{2}\left(K_{2323}-K_{1324}\right), \\
& B_{1,3}=\frac{U_{0}}{2}\left(K_{1214}-K_{2213}\right), \\
& B_{1,4}=\frac{U_{0}}{2}\left(K_{1434}-K_{2433}\right), \\
& B_{1,5}=-\frac{1}{2}-\frac{U_{0}}{2}\left(K_{1144}-K_{2143}\right), \\
& B_{1,6}=\frac{U_{0}}{2}\left(K_{1344}-K_{2343}\right), \\
& B_{1,7}=\frac{U_{0}}{2}\left(K_{1444}+K_{2223}-K_{1224}-K_{2443}\right), \\
& B_{1,8}=\frac{U_{0}}{2}\left(K_{1334}+K_{2113}-K_{1114}-K_{2333}\right), \\
& B_{21}=\frac{U_{0}}{2}\left(K_{3122}-K_{4121}\right),
\end{aligned}
$$




$$
\begin{aligned}
& B_{2,2}=-\frac{1}{2}-\frac{U_{0}}{2}\left(K_{3322}-K_{4321}\right) \text {, } \\
& B_{2,3}=\frac{U_{0}}{2}\left(K_{3212}-K_{4211}\right) \text {, } \\
& B_{2,4}=\frac{U_{0}}{2}\left(K_{3432}-K_{4431}\right), \\
& B_{2,5}=\frac{U_{0}}{2}\left(K_{4141}-K_{3142}\right) \text {, } \\
& B_{2,6}=\frac{U_{0}}{2}\left(K_{3342}-K_{4341}\right) \text {, } \\
& B_{2,7}=\frac{U_{0}}{2}\left(K_{3442}+K_{4221}-K_{3222}-K_{4441}\right) \text {, } \\
& B_{2,8}=\frac{U_{0}}{2}\left(K_{3332}+K_{4111}-K_{3112}-K_{4331}\right) \text {, } \\
& C_{1,1}=U_{0}\left(K_{1232}-K_{1142}\right), \\
& C_{1,2}=U_{0}\left(K_{1412}-K_{1322}\right) \text {, } \\
& C_{2,1}=U_{0}\left(K_{3232}-K_{3142}\right) \text {, } \\
& C_{2,2}=-1-U_{0}\left(K_{3322}-K_{3412}\right) \text {, } \\
& C_{3,1}=U_{0}\left(K_{2231}-K_{2141}\right) \text {, } \\
& C_{3,2}=U_{0}\left(K_{2411}-K_{2321}\right) \text {, } \\
& C_{4,1}=U_{0}\left(K_{4233}-K_{4143}\right) \text {, } \\
& C_{4,2}=U_{0}\left(K_{4413}-K_{4323}\right) \text {, } \\
& C_{5,1}=-1-U_{0}\left(K_{1144}-K_{1234}\right) \text {, } \\
& C_{5,2}=U_{0}\left(K_{1414}-K_{1324}\right) \text {, } \\
& C_{6,1}=U_{0}\left(K_{3234}-K_{3144}\right) \text {, } \\
& C_{6,2}=U_{0}\left(K_{3414}-K_{3324}\right) \text {, } \\
& C_{7,1}=\frac{U_{0}}{2}\left(K_{1141}+K_{3233}-K_{1231}-K_{3143}\right) \text {, } \\
& C_{7,2}=\frac{U_{0}}{2}\left(K_{1321}+K_{3413}-K_{1411}-K_{3413}\right) \text {, } \\
& C_{8,1}=\frac{U_{0}}{2}\left(K_{2142}+K_{4234}-K_{2232}-K_{4144}\right) \text {, } \\
& C_{8,2}=\frac{U_{0}}{2}\left(K_{2322}+K_{4414}-K_{2412}-K_{4324}\right) \text {, } \\
& D_{1,1}=1-U_{0} K_{1122} \text {, } \\
& D_{1,2}=U_{0} K_{1322} \text {, } \\
& D_{1,3}=-U_{0} K_{1212} \text {, } \\
& D_{1,4}=-U_{0} K_{1432} \text {, } \\
& D_{1,5}=U_{0} K_{1142} \text {, } \\
& D_{1,6}=-U_{0} K_{1342}, \\
& D_{1,7}=U_{0}\left(K_{1222}-K_{1442}\right) \text {, } \\
& D_{1,8}=U_{0}\left(K_{1112}-K_{1332}\right) \text {, } \\
& D_{2,1}=-U_{0} K_{3122} \text {, } \\
& D_{2,2}=1+U_{0} K_{3322} \text {, } \\
& D_{2,3}=-U_{0} K_{3212} \text {, } \\
& D_{2,4}=-U_{0} K_{3432} \text {, } \\
& D_{2,5}=U_{0} K_{3142} \text {, } \\
& D_{2,6}=-U_{0} K_{3342} \text {, } \\
& D_{2,7}=-U_{0}\left(K_{3222}-K_{3442}\right) \text {, } \\
& D_{2,8}=U_{0}\left(K_{3112}-K_{3332}\right) \text {, } \\
& D_{3,1}=-U_{0} K_{2121} \text {, } \\
& D_{3,2}=U_{0} K_{2321} \text {, } \\
& D_{3,3}=1-U_{0} K_{2211} \text {, } \\
& D_{3,4}=-U_{0} K_{2431} \text {, } \\
& D_{3,5}=U_{0} K_{2141} \text {, } \\
& D_{3,6}=-U_{0} K_{2341} \text {, } \\
& D_{3,7}=U_{0}\left(K_{2221}-K_{2441}\right) \text {, } \\
& D_{3,8}=U_{0}\left(K_{2111}-K_{2331}\right) \text {, } \\
& D_{4,1}=-U_{0} K_{4123} \text {, } \\
& D_{4,2}=U_{0} K_{4323} \text {, } \\
& D_{4,3}=-U_{0} K_{4213} \text {, } \\
& D_{4,4}=1-U_{0} K_{4433} \text {, } \\
& D_{4,5}=U_{0} K_{4143} \text {, } \\
& D_{4,6}=-U_{0} K_{4343} \text {, } \\
& D_{4,7}=U_{0}\left(K_{4223}-K 4443\right) \text {, } \\
& D_{4,8}=U_{0}\left(K_{4113}-K_{4333}\right) \text {, } \\
& D_{5,1}=-U_{0} K_{1124} \text {, } \\
& D_{5,2}=U_{0} K_{1324} \text {, } \\
& D_{5,3}=-U_{0} K_{1214} \text {, } \\
& D_{5,4}=-U_{0} \mathrm{~K}_{1434} \text {, } \\
& D_{5,5}=1+U_{0} K_{1144} \text {, } \\
& D_{5,6}=-U_{0} K_{1344} \text {, } \\
& D_{5,7}=U_{0}\left(K_{1224}-K 1444\right) \text {, }
\end{aligned}
$$




$$
\begin{aligned}
& D_{5,8}=U_{0}\left(K_{1114}-K_{1334}\right) \text {, } \\
& D_{6,1}=-U_{0} K_{3124} \text {, } \\
& D_{6,2}=U_{0} K_{3324} \text {, } \\
& D_{6,3}=-U_{0} K_{3214} \text {, } \\
& D_{6,4}=-U_{0} K_{3434} \text {, } \\
& D_{6,5}=U_{0} K_{3144} \text {, } \\
& D_{6,6}=1-U_{0} K_{3344} \text {, } \\
& D_{6,7}=U_{0}\left(K_{3224}-K 3444\right) \text {, } \\
& D_{6,8}=U_{0}\left(K_{3114}-K_{3334}\right) \text {, } \\
& D_{7,1}=\frac{U_{0}}{2}\left(K_{1121}-K_{3123}\right) \text {, } \\
& D_{7,2}=\frac{U_{0}}{2}\left(K_{3323}-K_{1321}\right) \text {, } \\
& D_{7,3}=\frac{U_{0}}{2}\left(K_{1211}-K_{3213}\right) \text {, } \\
& D_{7,4}=\frac{U_{0}}{2}\left(K_{1431}-K_{3433}\right) \text {, } \\
& D_{7,5}=\frac{U_{0}}{2}\left(K_{3143}-K_{1141}\right) \text {, } \\
& D_{7,6}=\frac{U_{0}}{2}\left(K_{1342}-K_{3343}\right) \text {, } \\
& D_{7,7}=-1-\frac{U_{0}}{2}\left(K_{1221}+K_{3443}-K_{1441}-K_{3223}\right) \text {, } \\
& D_{7,8}=\frac{U_{0}}{2}\left(K_{1331}+K_{3113}-K_{1111}-K_{3333}\right) \text {, } \\
& D_{8,1}=\frac{U_{0}}{2}\left(K_{2122}-K_{4124}\right) \text {, } \\
& D_{8,2}=\frac{U_{0}}{2}\left(K_{4324}-K_{2322}\right) \text {, } \\
& D_{8,3}=\frac{U_{0}}{2}\left(K_{2212}-K_{4214}\right) \text {, } \\
& D_{8,4}=\frac{U_{0}}{2}\left(K_{2432}-K_{4434}\right) \text {, } \\
& D_{8,5}=\frac{U_{0}}{2}\left(K_{4144}-K_{2142}\right) \text {, } \\
& D_{8,6}=\frac{U_{0}}{2}\left(K_{2342}-K_{4344}\right) \text {, } \\
& D_{8,7}=\frac{U_{0}}{2}\left(K_{2442}+K_{4224}-K_{2222}-K_{4444}\right) \\
& D_{8,8}=-1-\frac{U_{0}}{2}\left(K_{2112}+K_{4334}-K_{2332}-K_{4114}\right) \text {. }
\end{aligned}
$$

\section{Conflicts of Interest}

The authors declare that they have no conflicts of interest.

\section{Acknowledgments}

Israel Chávez Villalpando gratefully acknowledges funding from CONACyT Grant no. 291001 and UNAM-DGAPAPAPIIT IN102417.

\section{References}

[1] H. Pino, E. Alba, J. Taron, J. J. Garcia-Ripoll, and N. Barberán, "Hall response of interacting bosonic atoms in strong gauge fields: from condensed to fractional-quantum-Hall states," Physical Review A: Atomic, Molecular and Optical Physics, vol. 87, no. 5, Article ID 053611, 2013.

[2] N. Goldman, J. Beugnon, and F. Gerbier, "Detecting chiral edge states in the hofstadter optical lattice," Physical Review Letters, vol. 108, no. 25, Article ID 255303, 2012.

[3] N. Goldman, J. Beugnon, and F. Gerbier, "Identifying topological edge states in 2D optical lattices using light scattering," The European Physical Journal Special Topics, vol. 217, no. 1, pp. 135152, 2013.

[4] M. Greiner, C. A. Regal, J. T. Stewart, and D. S. Jin, "Probing pair-correlated fermionic atoms through correlations in atom shot noise," Physical Review Letters, vol. 94, no. 11, Article ID 110401, 2005.

[5] L. Tarruell, D. Greif, T. Uehlinger, G. Jotzu, and T. Esslinger, "Creating, moving and merging Dirac points with a Fermi gas in a tunable honeycomb lattice," Nature, vol. 483, no. 7389, pp. 302-305, 2012.

[6] Z. Fu, L. Huang, Z. Meng et al., "Radio-frequency spectroscopy of a strongly interacting spin-orbit-coupled Fermi gas," Physical Review A: Atomic, Molecular and Optical Physics, vol. 87, no. 5, Article ID 053619, 2013.

[7] L. He and X.-G. Huang, "Nonperturbative effects on the ferromagnetic transition in repulsive Fermi gases," Physical Review A: Atomic, Molecular and Optical Physics, vol. 85, no. 4, Article ID 043618, 2012.

[8] L. He and X.-G. Huang, "Superfluidity and collective modes in Rashba spin-orbit coupled Fermi gases," Annals of Physics, vol. 337, pp. 163-207, 2013.

[9] Y. Xu, R.-L. Chu, and C. Zhang, "Anisotropic weyl fermions from the quasiparticle excitation spectrum of a 3D fulde-ferrell superfluid," Physical Review Letters, vol. 112, no. 13, Article ID 136402, 2014.

[10] I. I. Satija, D. C. Dakin, J. Y. Vaishnav, and C. W. Clark, "Physics of a two-dimensional electron gas with cold atoms in nonAbelian gauge potentials," Physical Review A: Atomic, Molecular and Optical Physics, vol. 77, no. 4, Article ID 043410, 2008.

[11] A. Bermudez, N. Goldman, A. Kubashiak, M. Lewenstein, and M. A. Martin-Delgado, "Non-abelian optical lattices: anomalous quantum hall effect and dirac fermions," Physical Review Letters, vol. 103, Article ID 035301, 2009.

[12] A. Bermudez, N. Goldman, A. Kubasiak, M. Lewenstein, and M. A. Martin-Delgado, "Topological phase transitions in the non-Abelian honeycomb lattice," New Journal of Physics, vol. 12, Article ID 033041, 2010.

[13] N. Goldman, A. Kubasiak, P. Gaspard, and M. Lewenstein, "Ultracold atomic gases in non-Abelian gauge potentials: the 
case of constant Wilson loop," Physical Review A: Atomic, Molecular and Optical Physics, vol. 79, no. 2, Article ID 023624, 2009.

[14] A. Kubasiak, P. Massignan, and M. Lewenstein, "Topological superfluids on a lattice with non-Abelian gauge fields," $E P L$ (Europhysics Letters), vol. 92, no. 4, Article ID 46004, 2010.

[15] L. Wang and L. Fu, "Interaction-induced merging of Dirac points in non-Abelian optical lattices," Physical Review A: Atomic, Molecular and Optical Physics, vol. 87, no. 5, Article ID 053612, 2013.

[16] M. Iskin, "Superfluid phases of ultracold Fermi gases on a checkerboard superlattice," Physical Review A: Atomic, Molecular and Optical Physics, vol. 88, no. 5, Article ID 013631, 2013.

[17] M. Burrello, I. C. Fulga, E. Alba, L. Lepori, and A. Trombettoni, "Topological phase transitions driven by non-Abelian gauge potentials in optical square lattices," Physical Review A: Atomic, Molecular and Optical Physics, vol. 88, no. 5, Article ID 053619, 2013.

[18] Q. Sun, G.-B. Zhu, W.-M. Liu, and A.-C. Ji, "Spin-orbit coupling effects on the superfluidity of a Fermi gas in an optical lattice," Physical Review A: Atomic, Molecular and Optical Physics, vol. 88, no. 6, Article ID 063637, 2013.

[19] Y. Zhang and C. Zhang, "Bose-einstein condensates in spinorbit-coupled optical lattices: flat bands and superfluidity," Physical Review A, vol. 87, Article ID 023611, 2013.

[20] F. Lin, C. Zhang, and V. W. Scarola, "Emergent kinetics and fractionalized charge in 1D spin-orbit coupled flatband optical lattices," Physical Review Letters, vol. 112, no. 11, Article ID 110404, 2014.

[21] D. Toniolo and J. Linder, "Superfluidity breakdown and multiple roton gaps in spin-orbit-coupled Bose-Einstein condensates in an optical lattice," Physical Review A: Atomic, Molecular and Optical Physics, vol. 89, no. 6, Article ID 061605(R), 2014.

[22] C. Qu, M. Gong, and C. Zhang, "Fulde-Ferrell-Larkin-Ovchinnikov or Majorana superfluids: the fate of fermionic cold atoms in spin-orbit-coupled optical lattices," Physical Review A, vol. 89, Article ID 053618, 2014.

[23] Y. Xu, C. Qu, M. Gong, and C. Zhang, “Competing superfluid orders in spin-orbit-coupled fermionic cold-atom optical lattices," Physical Review A, vol. 89, Article ID 013607, 2014.

[24] H. Zhai, "Degenerate quantum gases with spin-orbit coupling: a review," Reports on Progress in Physics, vol. 78, no. 2, Article ID 026001, 25 pages, 2015.

[25] H.-K. Tang, X. Yang, J. Sun, and H.-Q. Lin, "BerezinskiiKosterlitz-Thoules phase transition of spin-orbit coupled Fermi gas in optical lattice," Europhysics Letters, vol. 107, Article ID 40003, 2014.

[26] M. Iskin, "Topological superfluids on a square optical lattice with non-Abelian gauge fields: effects of next-nearest-neighbor hopping in the BCS-BEC evolution," Physical Review A: Atomic, Molecular and Optical Physics, vol. 93, Article ID 013608, 2016.

[27] C. Itzykson and J. B. Zuber, Quantum Field Theory, McGrawHill, New York, NY, USA, 1980.

[28] J. Schwinger, "The theory of quantizied fields," Physical Review A: Atomic, Molecular and Optical Physics, vol. 82, p. 914, 1951.

[29] F. J. Dyson, "The $S$ matrix in quantum electrodynamics," Physical Review A: Atomic, Molecular and Optical Physics, vol. 75, no. 11, pp. 1736-1755, 1949.

[30] H. A. Bethe and E. E. Salpeter, "A relativistic equation for bound-state problems," Physical Review A: Atomic, Molecular and Optical Physics, vol. 82, p. 309, 1951.
[31] H. A. Bethe and E. E. Salpeter, "A relativistic equation for bound-state problems," Physical Review A: Atomic, Molecular and Optical Physics, vol. 84, p. 1232, 1951.

[32] Z. G. Koinov, "Collective modes of an ultracold ${ }^{6} \mathrm{Li}^{4}{ }^{40} \mathrm{~K}$ mixture in an optical lattice," Advances in Condensed Matter Physics, vol. 2015, Article ID 952852, 12 pages, 2015. 

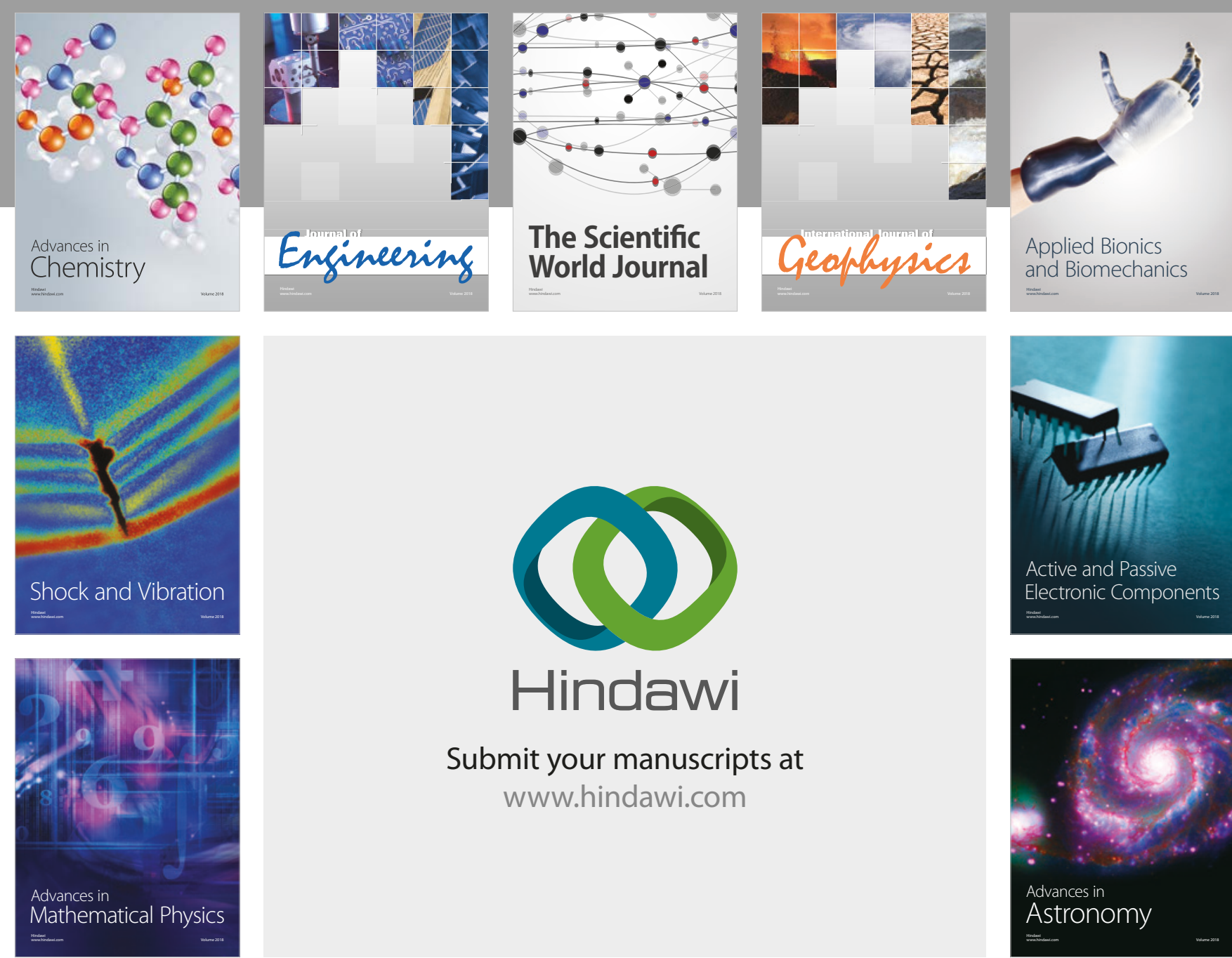

Submit your manuscripts at

www.hindawi.com

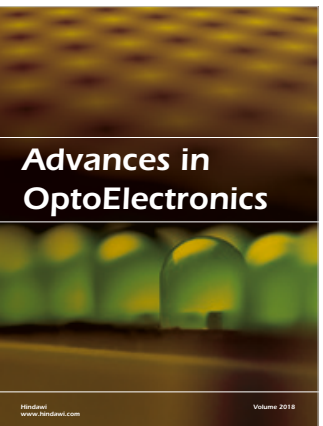

\section{Rotcting Machinery}
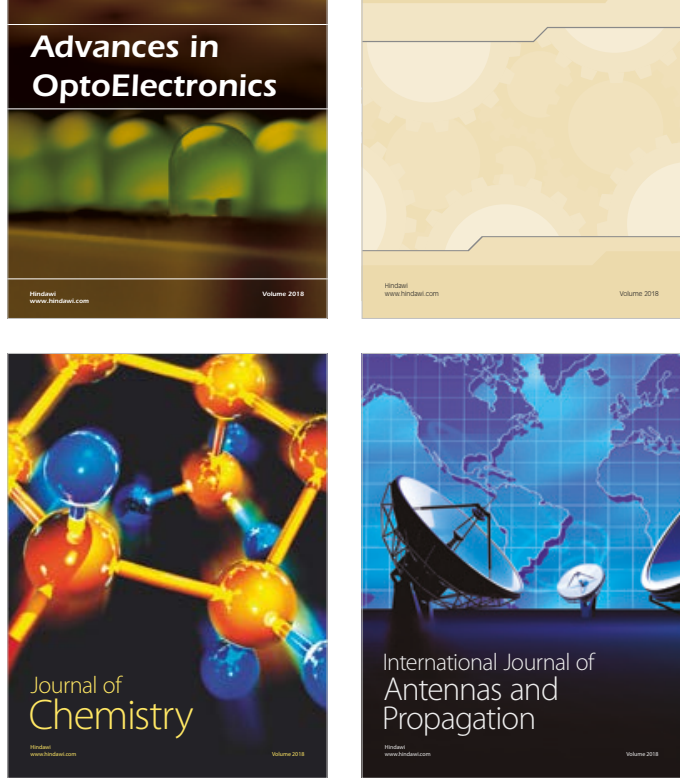

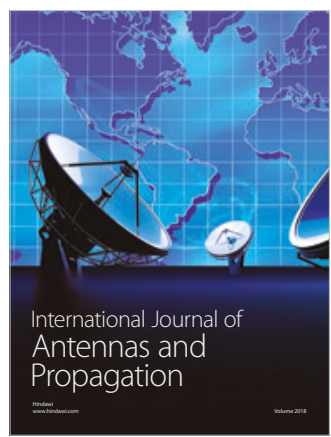

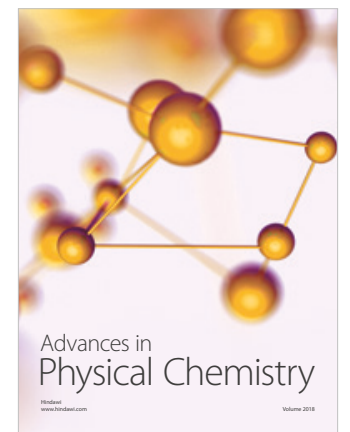

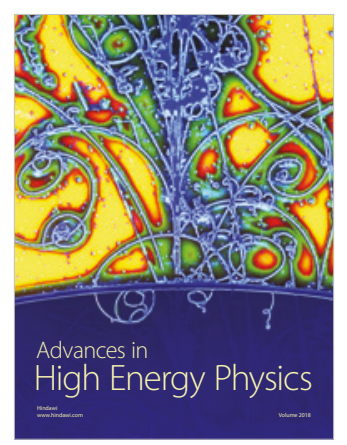

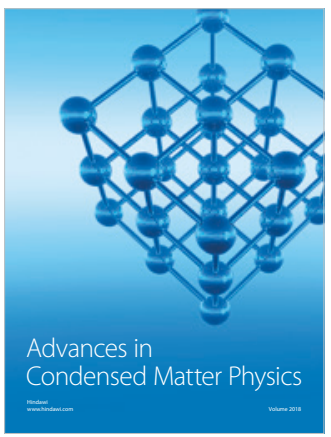

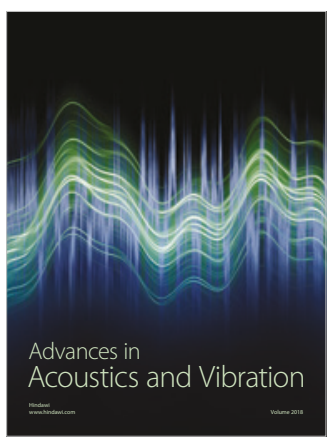

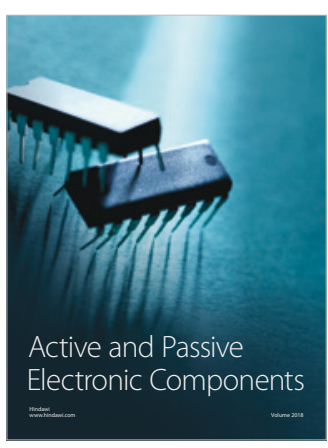
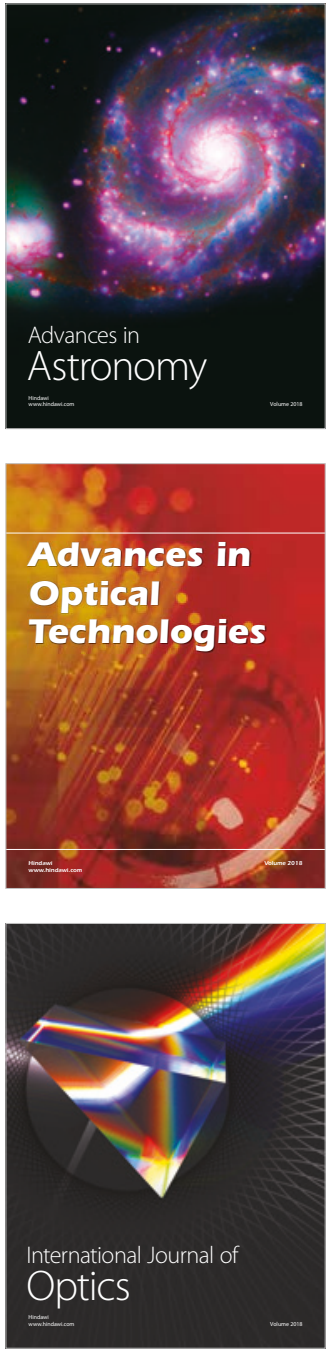